\title{
Qualidade da Pesquisa Científica Brasileira em Informática na Educação: Desafios e Perspectivas
}

\author{
Patricia A. Jaques $^{1}$, Sean Siqueira ${ }^{2}$, Ig I. Bittencourt ${ }^{3}$, Seiji Isotani ${ }^{4}$ \\ ${ }^{1}$ PIPCA - Universidade do Vale do Rio dos Sinos (UNISINOS) \\ ${ }^{2}$ Universidade Estadual do Rio de Janeiro (UNIRIO) \\ ${ }^{3}$ Universidade Federal de Alagoas (UFAL) \\ ${ }^{4}$ ICMC - Universidade do Estado de São Paulo (USP) \\ pjaques@unisinos.br, sean@uniriotec.br \\ ig.iberteic.ufal.br, sisotanidicmc.usp.br
}

\begin{abstract}
This article aims to discuss the challenge of the quality of the Brazilian scientific research in Computers in Education. Initially, we present the current research scenario as a way of identifying factors that determine the quality and national and international impact of the research in the area. Afterwards, we propose some actions to be accomplished by the Brazilian Special Commission on Computers in Education to promote improvements. These actions are organized in four axes: (i) Curate a history of the area of Computers in Education in Brazil, including public policy initiatives and stories of success and failure; (ii) Rationale of the concepts and terminologies of the area of Computers in Education; (iii) Advancement and Dissemination of the state of the art and practice in the area of Computers in Education; (iv) Dissemination and evolution of research methods in Computers in Education. Finally, to verify the impact of the proposed actions on the quality of Brazilian research in Computers in Education, we propose some metrics, which will allow a quantitative and objective analysis of the impact of the proposed actions.
\end{abstract}

Resumo. O presente artigo busca discutir o desafio da qualidade da pesquisa científica em informática na educação no Brasil. Inicialmente, apresenta-se o cenário atual da pesquisa como forma de identificar fatores que determinam a qualidade e impacto nacional e internacional da pesquisa na área. Após identificação desses fatores, são apresentadas ações a serem realizadas pela comunidade e coordenadas pela Comissão Especial de Informática na Educação a fim de promover melhorias. Essas ações estão organizadas em quatro eixos: (i) Curadoria de um histórico da área de Informática na Educação no Brasil, incluindo iniciativas de políticas públicas e histórias de sucesso e fracasso; (ii) Fundamentação da área de Informática na Educação; (iii) Avanço e disseminação do estado da arte e da prática na área de Informática na Educação; (iv) Disseminação e evolução de métodos de pesquisa em Informática na Educação. Por último, para verificar o impacto das ações propostas na qualidade da pesquisa brasileira na área de IE, o artigo também propõe métricas, o que possibilitará uma análise quantitativa e objetiva das ações propostas. 


\section{Introdução}

A pesquisa científica brasileira tem motivos para comemorar. Nas três últimas décadas, o país teve seu número de artigos científicos publicados ampliado. Passou de 2.000 artigos por ano em 1981 para $40.500 \mathrm{em} 2014$ [Oliveira 2016]. Este resultado fez com que a posição mundial do Brasil subisse de $23^{\circ}$ para $13^{\circ}$, em se tratando de número de artigos publicados, posicionando o país como líder da américa latina [Cruz 2013].

No entanto, esse papel central contrasta com o baixo impacto (número de citações) e visibilidade (publicações em periódicos de prestígio internacional) das publicações científicas brasileiras [Leta et al. 2013], que chega a ser menor do que alguns países latinoamericanos [Oliveira 2016]. Além disso, a ciência brasileira tem sido também criticada pela sua baixa eficiência [Leite 2015], ou seja, o número de artigos publicados em periódicos de alto prestígio internacional é muito baixo em relação ao investimento realizado em pesquisa [Volpato 2015].

Mas como melhorar o impacto das publicações científicas? A publicação de trabalhos de grande impacto se deve a fatores como escolha de problemas científicos com apelo, cientistas altamente qualificados, infraestrutura adequada e habilidade de comunicar os resultados científicos [Oliveira 2016, Volpato 2015].

$\mathrm{Na}$ área de Informática na Educação (IE) não é diferente. Para que os pesquisadores apresentem resultados científicos inovadores e de reconhecida qualidade científica internacional é primordial que os autores conheçam o histórico e tenham uma boa fundamentação da área, estando ainda por dentro do estado da arte e da prática em IE. É, sobretudo, primordial que os trabalhos sigam rigorosa metodologia científica, propondo questões de pesquisa para problemas originais e atuais e passando por um processo de avaliação empírica que busque objetivamente mostrar o impacto da abordagem proposta na aprendizagem, direta ou indiretamente, objetivo final da área de pesquisa em IE.

Observa-se, no entanto, que embora a IE tenha mais de 40 anos no Brasil, muitas pesquisas desenvolvidas na área ainda possuem problemas conceituais, apresentação superficial e inadequada do estado da arte e/ou da prática, bem como algumas limitações metodológicas. O Simpósio Brasileiro de Informática na Educação (SBIE) é a principal conferência nacional na área. Em uma revisão sistemática organizada por Magalhães et al. [Magalhães et al. 2013] dos artigos publicados neste simpósio, verificou-se que, dos 835 artigos publicados entre os anos de 2000 a 2010, apenas $29 \%$ apresentaram alguma avaliação empírica, seja ela qualitativa (estudo de caso, pesquisa-ação etc) ou quantitativa (experimentos ou quasi-experimentos), embora, os autores relatem um aumento progressivo da quantidade de artigos com avaliações nas últimas edições do evento.

A Comissão Especial de Informática na Educação (CEIE) ${ }^{1}$ da SBC tem buscado entender o porquê desses resultados. Uma primeira questão é que embora a área de pesquisa de IE seja inerentemente inter/transdisciplinar, a pesquisa em informática na educação acontece essencialmente em Programas de Pós-Graduação (PPGs) que são voltados a áreas específicas. Por exemplo, geralmente, as universidades brasileiras possuem PPGs em Computação e/ou Educação isolados. Esses programas são avaliados essencialmente pelas qualidades das publicações, segundo o sistema Qualis, que possui classificação por área, atendendo a critérios específicos desta área. Esse cenário não in-

\footnotetext{
${ }^{1}$ http: //ceie-br.org
} 
centiva o desenvolvimento conjunto de pesquisa entre pesquisadores de áreas diferentes. Tentando minimizar esse problema, o Qualis conta atualmente com a área Interdisciplinar. No entanto, os conceitos dessa área são válidos apenas para PPGs enquadrados nela. Os proponentes estão cientes de um único PPG interdisciplinar brasileiro voltado exclusivamente à IE, o PPGIE da UFRGS. Embora o tema do maior congresso da Sociedade Brasileira de Computação (CSBC) no ano de 2016 tenha sido interdisciplinaridade, e o tema tenha sido mais amplamente discutido na Computação no Brasil nos últimos anos, poucos resultados concretos têm sido vistos no sentido de promover a pesquisa interdisciplinar no Brasil. Dados os desafios da interdisciplinaridade, é essencial que os pesquisadores da área entendam os desafios já enfrentados (histórico), bem como a fundamentação de diferentes conceitos, técnicas, ferramentas etc. de cada área relacionada.

Além disso, uma outra lacuna que se observa é a ausência de materiais específicos para metodologia de pesquisa em Informática na Educação. Realizar pesquisa é mais do que seguir as etapas de um programa de pós-graduação (projeto, exame de qualificação, defesa, entre outros). Ela envolve uma série de métodos que devem ser aplicados de forma crítica e analítica para se gerar um conhecimento científico novo. Isso é importante para que a comunidade avance globalmente em relação aos trabalhos no formato "TCC-like", que envolvem "uma proposta de um sistema para "alguma coisa" [Wazlawick 2009].

A problemática da interdisciplinaridade, já levantada, também dificulta, além da fundamentação na área, a produção do material para metodologia de pesquisa na área, uma vez que a Informática na Educação engloba métodos de pesquisa de diferentes áreas, como computação, psicologia, educação, entre outros. Observa-se inclusive a escassez de materiais na literatura internacional [Mark and Greer 1993, Greer and Mark 2016]. Não menos importante, a formação dos futuros pesquisadores deve envolver formação específica em escrita científica, como apontado por Oliveira (2016).

Nesse contexto, os autores se perguntaram que ações poderiam ser coordenadas pela CEIE para ajudar a melhorar a qualidade da pesquisa em Informática na Educação no Brasil. As ações propostas estão classificadas em quatro categorias:

I) Curadoria de um histórico da área de Informática na Educação no Brasil, incluindo iniciativas de políticas públicas e histórias de sucesso e ou fracasso: a comunidade precisa conhecer seu histórico para aprender com erros e acertos passados e trabalhar de forma a avançar incrementalmente a ciência.

II) Fundamentação da área de Informática na Educação: conhecer os fundamentos das teorias de áreas que fundamentam a IE é essencial para realizar pesquisa com qualidade, baseada em background teórico sólido.

III) Avanço e disseminação do estado da arte e da prática na área de Informática na Educação: conhecer o estado da arte é essencial para propor problemas científicos atuais e originais. $\mathrm{O}$ trabalho do pesquisador seria facilitado se esse conhecimento já estiver organizado de maneira sistemática.

IV) Disseminação e evolução de métodos de pesquisa em Informática na Educação: o conhecimento dos métodos científicos é essencial para seu emprego adequado, de modo a propiciar a validação da pesquisa e, portanto, o desenvolvimento de pesquisa de qualidade.

As ações classificadas de acordo com as categorias supramencionadas são descritas na Seção 2. Para verificar o impacto das ações propostas na qualidade da pesquisa 
brasileira na área de IE, propomos métricas, descritas na Seção 3, o que possibilitará uma análise quantitativa e objetiva dos resultados alcançados.

\section{Ações para melhoria da qualidade da pesquisa brasileira em Informática na Educação}

Com o objetivo de melhorar a qualidade da pesquisa na área de informática na educação, propomos algumas ações a serem assumidas pela comunidade de IE no Brasil e coordenadas pela CEIE. É importante salientar que algumas dessas ações já vêm sendo realizadas pela CEIE, bem como outras soluções ainda podem surgir, evoluindo a proposta original à medida que a comunidade apoie e as tecnologias se desenvolvam. Essas ações estão organizadas em quatro categorias, conforme descrito a seguir.

I) A primeira categoria envolve a realização de uma curadoria de um histórico da área de Informática na Educação no Brasil, incluindo iniciativas de políticas públicas (por exemplo, um computador por aluno) e histórias de sucesso e fracasso. A ideia é que esse histórico seja mantido em um portal web multimídia que organizaria a memória da área no Brasil. As seguintes ações são previstas dentro dessa categoria:

- Entrevistar personagens históricos da comunidade de pesquisa em IE. Aqui incluímos profissionais conhecidos por serem pioneiros na área de IE no Brasil ou por terem promovido eventos, periódicos ou outras ações importantes na área.

- Fazer levantamento do histórico das conferências e periódicos mantidos pela CEIE.

- Preparar um survey a ser divulgado na comunidade brasileira de IE para levantar as principais ações brasileiras em pesquisa, assim como políticas públicas na área de IE.

- Levantar dados adicionais das ações identificadas no item anterior através de entrevistas, e-mails e acesso a web.

- Organizar de maneira consistente e contextualizada as informações coletadas.

- Disponibilizar as informações do histórico em um portal web multimídia, por exemplo, o próprio portal da CEIE.

- Promover mecanismos de armazenamento, atualização, recuperação, visualização e disseminação do material.

II) A segunda categoria envolve realizar uma fundamentação da área de Informática na Educação. O principal objetivo aqui é organizar e manter um repositório evolutivo de conceitos, técnicas, procedimentos, tecnologias etc. importantes para a área de pesquisa em IE no Brasil e suas interseções com o que é realizado internacionalmente. Essa categoria envolveria ações como:

- Organização dos principais conceitos e terminologia da área em uma ontologia.

- Organização das teorias relacionadas a ensino e aprendizagem em um repositório multimídia.

- Organização de um repositório multimídia (portal web) de técnicas e procedimentos de como utilizar informática para apoiar o ensino-aprendizagem.

- Edição de um livro texto na área de Informática na Educação. 
- Organização de um portfólio de tecnologias de mercado (e seus fornecedores) utilizadas para apoiar o ensino-aprendizagem.

III) A terceira categoria envolve o avanço e disseminação do estado da arte e da prática na área de Informática na Educação. A principal ideia aqui é criar ferramentas que permitam à comunidade de pesquisa em Informática na Educação se atualizar tanto teoricamente quanto tecnologicamente do estado da arte e da prática da área. Essa categoria engloba ações como:

- Criação de um portal colaborativo para os pesquisadores compartilharem suas experiências de pesquisa teórica e tecnológica em Informática na Educação.

- Criação de um repositório de aplicações na área de informática na educação, para que os pesquisadores possam disseminar suas soluções e disponibilizar suas aplicações, incentivando principalmente a filosofia de código e dados abertos.

- Criação de um repositório de conteúdos (abertos) integrador, que permita a busca, compartilhamento e reuso de conteúdos, com a devida acessibilidade, personalização e adaptação, com base na filosofia de dados abertos.

- Divulgação e disseminação das publicações da área de IE no Brasil, buscando aumentar sua visibilidade.

- Organização de um banco de experiências do uso de Informática na Escola para promover a disseminação de boas e más práticas, bem como o compartilhamento de experiências e seus contextos, considerando as diversas facetas envolvidas.

IV) A quarta categoria visa a disseminação e evolução de métodos de pesquisa em Informática na Educação. Essa categoria englobaria ações como:

- Edição de um livro voltado à Metodologia de Pesquisa em Informática na Educação e Escrita Científica.

- Catálogo de métodos de pesquisa com exemplos, a ser disponibilizados em um portal da comunidade, complementando o livro.

- Promoção de Painéis para discussão sobre Qualidade da Pesquisa em Informática na Educação nos principais eventos na área (por ex., DesafIE e CBIE).

- Proposta de eventos e cursos específicos, tais como minicursos (por exemplo, JAIE) e Escolas de Verão, voltados à comunidade e que busquem promover uma formação mais multidisciplinar em qualidade na pesquisa em informática na educação, assim como desenvolver nos jovens pesquisadores o pensamento crítico, lógico e analítico-científico e a cultura da qualidade da pesquisa.

- Análise automática de artigos publicados nos principais veículos nacionais de divulgação científica para captura de métodos utilizados.

- Geração de guidelines para avaliadores dos eventos da CEIE.

- Criação de repositórios de dados (benchmarks). Os benchmarks consistem de base de dados para avaliação de ferramentas de um domínio (por exemplo, benchmarks de expressões faciais emocionais para avaliação de ferramentas de detecção de emoções). Eles permitiriam comparar as diferentes soluções com os mesmos dados, o que levaria a uma evolução natural destas. 


\section{Métricas para avaliar o impacto das ações}

Por último, para verificar o impacto das ações propostas na qualidade da pesquisa brasileira na área de IE, propomos algumas métricas, o que possibilitará uma análise quantitativa e objetiva dos resultados alcançados com as ações propostas. Essas métricas visam avaliar o impacto das ações propostas neste artigo para a promoção da qualidade da pesquisa na área de Informática na Educação. São elas:

- Verificar se houve aumento do número de artigos publicados com avaliação empírica nas principais conferências e periódicos na área;

- Verificar se houve aumento no número de pesquisadores na área de IE com bolsa de produtividade. Importante observar que essa métrica vai depender de políticas de órgãos de fomento que podem não aumentar o número de bolsas ou até reduzilas, o que tornaria essa métrica inaplicável. No entanto, caso o número de bolsas permaneça constante, poderá ainda ser observado se, na área da computação e educação, houve migração das bolsas que estavam alocadas para pesquisadores de outras subáreas para pesquisadores de IE;

- Verificar se houve aumento do índice de impacto (e.g. número de citações, geração de políticas educacionais baseada em dados publicados etc.) dos principais veículos de divulgação científica mantidos pela CEIE;

- Verificar se houve aumento do impacto das publicações internacionais na área de IE realizada por brasileiros.

Nossa ideia é que essas métricas sejam aplicadas em um estudo longitudinal, ou seja, em vários momentos nos próximos 10 anos. Isso nos permitiria observar a variação histórica dos dados obtidos pelas métricas, o que possibilitaria ter mais confiança na evolução dos dados, se for o caso, e também avaliar ações que possivelmente tiveram um impacto maior. Por exemplo, se for observada uma evolução mais acentuada nos dados provenientes de uma ou várias métricas, que estavam estáveis, após uma determinada ação, isso pode ser um indício de que aquela ação teve um impacto maior.

\section{Conclusão}

A pesquisa brasileira fez avanços significativos nas últimas três décadas em relação ao número de artigos publicados, se posicionando como líder na américa latina. No entanto, em se tratando do impacto e visibilidade dessas publicações, assim como da eficiência do investimento em pesquisa, o Brasil está atrás de até mesmo vários países latinoamericanos [Oliveira 2016, Volpato 2015]. Essa situação não é diferente na comunidade de pesquisa em Informática na Educação, cujos artigos nos principais veículos de divulgação científica nacionais mostram falhas metodológicas [Magalhães et al. 2013]. A questão é então como aumentar o impacto das publicações científicas brasileiras na área de IE?

Para que a publicação tenha impacto é essencial que apresente resultados científicos inovadores e de reconhecida qualidade científica internacional. Para tanto, é primordial que os trabalhos sigam rigorosa metodologia científica, propondo questões de pesquisa para problemas originais e atuais e passando por um processo de avaliação empírica que busque objetivamente mostrar se os objetivos foram atingidos.

Assim, acreditamos que melhorar o impacto da qualidade da pesquisa em IE é essencial para melhorarmos o seu impacto internacional e também para melhorar a sua 
contribuição real à educação brasileira. Esse é um desafio que somente pode ser resolvido através de ações que busquem promover a qualidade científica na comunidade brasileira de pesquisadores. Dessa forma, propomos algumas ações que acreditamos que podem ser promovidas no âmbito da CEIE, com apoio da comunidade de IE no Brasil, de forma a criar uma cultura de valorização da qualidade da pesquisa, assim como promover o conhecimento conceitual e metodológico científico aplicado à área de Informática na Educação. Apenas com o esforço conjunto de amadurecimento da pesquisa em IE no Brasil, conseguiremos efetivamente promover a democratização da Informática na Educação, bem como ter impacto e relevância no cenário internacional.

\section{Referências}

Cruz, C. H. B. (2013). A busca pela excelência nas universidades no Brasil. http://www. fapesp.br/eventos/2014/01/Excellence/ excellence-in-higher-ed-chbc20140122.pdf. Acessado em 28 de fevereiro de 2017.

Greer, J. and Mark, M. A. (2016). Evaluation Methods for Intelligent Tutoring Systems Revisited. International Journal of Artificial Intelligence in Education, 26(1):387-392.

Leite, R. C. C. (2015). Produção científica e lixo acadêmico no Brasil. Folha de São Paulo, jan., A3, http://www1.folha.uol.com.br/fsp/opiniao/ 202892-producao-cientifica-e-lixo-academico-no-brasil. shtml. Acessado em 6 de abril de 2017.

Leta, J., Thijs, B., and Glänzel, W. (2013). A macro-level study of science in Brazil: seven years later. Encontros Bibli: revista eletrônica de biblioteconomia e ciência da informação, 18(36).

Magalhães, C. V. C., Santos, R. E., Da Silva, F. Q. B., and Gomes, A. S. (2013). Caracterizando a Pesquisa em Informática na Educação no Brasil: Um Mapeamento Sistemático das Publicações do SBIE. In XXIV Simpósio Brasileiro de Informática na Educação. SBC/CEIE.

Mark, M. A. and Greer, J. E. (1993). Evaluation methodologies for intelligent tutoring systems. Journal of Artificial Intelligence and Education, 4(2/3):129-153.

Oliveira, O. N. (2016). Research Landscape in Brazil: Challenges and Opportunities. The Journal of Physical Chemistry C, 120(10):5273-5276.

Volpato, G. (2015). Guia Prático Para Redação Científica. Best Writing, 1 edition.

Wazlawick, R. S. (2009). Metodologia de Pesquisa para Ciência da Computação. Campus. 